\title{
RINGS IN WHICH CERTAIN SUBSETS SATISFY POLYNOMIAL IDENTITIES
}

\author{
BY \\ THOMAS P. KEZLAN
}

Let $R$ be an associative ring and $\mathscr{F}$ a set of polynomials (each in some finite number of noncommuting indeterminates) with integer coefficients. $R$ will be called an $\mathscr{F}$-ring if and only if for every finite subset $S$ of $R$ there is an $f$ in $\mathscr{F}$ which vanishes identically on $S$. Thus certain kinds of rings can be characterized as $\mathscr{F}$ rings for appropriate choices of $\mathscr{F}$ : nil rings, with $\mathscr{F}$ the set of polynomials $x^{n}$ for all positive integers $n$; locally nilpotent rings, with $\mathscr{F}$ the set of monomials $x_{1} x_{2} \cdots x_{n}$ in $n$ indeterminates for all $n$; nilpotent rings, with $\mathscr{F}$ the single monomial $x_{1} x_{2} \cdots x_{n}$ for some $n$; rings satisfying a polynomial identity, with $\mathscr{F}$ finite (or equivalently, with $\mathscr{F}$ containing a single polynomial); and so forth. In addition to $\mathscr{F}$-rings we shall consider rings satisfying a somewhat stronger property. $R$ is called an $\mathscr{F}^{+}$-ring if and only if for every finitely generated additive subgroup $S$ of $R$ there is an $f$ in $\mathscr{F}$ which vanishes identically on $S$. Clearly every $\mathscr{F}^{+}$-ring is an $\mathscr{F}$-ring. Also all subrings and homomorphic images of $\mathscr{F}$-rings are $\mathscr{F}$-rings, and similarly for $\mathscr{F}^{+}$-rings. This paper is concerned with determining conditions on $\mathscr{F}$ which will ensure that every $\mathscr{F}$-ring (or $\mathscr{F}^{+}$-ring) has nil commutator ideal (the ideal generated by all commutators $[a, b]=a b-b a$ ). This intent is similar to that of Drazin in [5] insofar as both are concerned with conditions under which, in a given class of rings, the fact that the commutator ideal is contained in the Jacobson radical implies that the commutator ideal is nil. Drazin's work is self-contained (depending only on Zorn's lemma) but requires that every ring in the class be an " $f$-ring" (cf. [5]), whereas here, rather than imposing conditions on our rings individually, we instead confine our attention to classes defined in special ways, i.e., as complete classes of $\mathscr{F}$ - or $\mathscr{F}^{+}$-rings, and make use of some relatively special properties of the Jacobson radical (whereas Drazin's arguments would apply equally well to any radical not containing nonzero idempotents).

The commutator ideal of $R$ is denoted $C(R)$ and the Jacobson radical $J(R)$. The ring of $n \times n$ matrices over $R$ is denoted $R_{n}$. $R$ is said to be of characteristic 0 , written char $(R)=0$, if and only if $m x \neq 0$ for every nonzero integer $m$ and every nonzero $x$ in $R$. A linear polynomial means a polynomial which is linear in each of its indeterminates; $\mathscr{F}$ will be called linear if and only if each member of $\mathscr{F}$ is linear. We consider only sets $\mathscr{F}$ of polynomials each of which is homogeneous in each of its indeterminates. One final assumption about $\mathscr{F}$ : Note that if all coeffi-

Presented to the Society, June 29, 1965; received by the editors August 3, 1965 and, in revised form, March 11, 1966. 
cients appearing in some member of $\mathscr{F}$ are divisible by the same prime $p$, then any ring of characteristic $p$ is trivially an $\mathscr{F}$-ring. To avoid this situation, we make the assumption that every member of $\mathscr{F}$ has the integer 1 for at least one of its coefficients.

Let $f$ be a polynomial in (noncommuting) indeterminates $x_{1}, x_{2}, \ldots, x_{n}$ with integer coefficients. We denote by $\hat{f}$ the linear polynomial obtained from $f$ by the usual "linearization" process [10, p. 224], and [11, Lemma 2]. That is, if $f$ is nonlinear in $x_{1}$, say of degree $d>1$, define

(1) $f_{1}\left(y, z, x_{2}, \ldots, x_{n}\right)=f\left(y+z, x_{2}, \ldots, x_{n}\right)-f\left(y, x_{2}, \ldots, x_{n}\right)-f\left(z, x_{2}, \ldots, x_{n}\right)$.

$f_{1}$ is then a polynomial in $n+1$ indeterminates and of degree $d-1$ in both $y$ and $z$. This process leads to a sequence of polynomials $f=f_{0}, f_{1}, \ldots, f_{k}=\hat{f}$, in which each $f_{i}$ is obtained by "linearizing" $f_{i-1}$ in some indeterminate by the process (1). For a given set $\mathscr{F}$ of polynomials let $\hat{\mathscr{F}}=\{\hat{f} \mid f \in \mathscr{F}\}$.

LEMMA 1. (i) Every $\mathscr{F}^{+}$-ring is an $\hat{\mathscr{F}}_{\text {-ring. }}$

(ii) Every $\hat{F}$-ring of characteristic 0 is an $\mathscr{F}^{+}$-ring (and hence an $\mathscr{F}$-ring).

Proof. (i) follows easily from the "linearization" process. Let $R$ be an $\hat{F}$-ring of characteristic 0 and $S$ a finitely generated additive subgroup of $R$. There exists $f$ in $\mathscr{F}$ such that $\hat{f}$ vanishes on the generators of $S$ and hence on $S$. Inductively, suppose that in the sequence $f=f_{0}, f_{1}, \ldots, f_{k}=\hat{f}$ obtained by "linearization" of $f, f_{i}$ vanishes on $S$, where $0<i \leqq k$. If $f_{i-1}$ is a polynomial in $r$ indeterminates $x_{1}, \ldots, x_{r}$, then without loss of generality, for some indeterminate $x_{r+1}$,

$f_{i}\left(x_{1}, \ldots, x_{r}, x_{r+1}\right)=f_{i-1}\left(x_{1}, \ldots, x_{r}+x_{r+1}\right)-f_{i-1}\left(x_{1}, \ldots, x_{r}\right)-f_{i-1}\left(x_{1}, \ldots, x_{r+1}\right)$.

Let $a_{1}, \ldots, a_{r}$ be any $r$ elements in $S$. Then

$$
\begin{aligned}
0 & =f_{i}\left(a_{1}, \ldots, a_{r}, a_{r}\right)=f_{i-1}\left(a_{1}, \ldots, 2 a_{r}\right)-2 f_{i-1}\left(a_{1}, \ldots, a_{r}\right) \\
& =\left(2^{d}-2\right) f_{i-1}\left(a_{1}, \ldots, a_{r}\right),
\end{aligned}
$$

where $d>1$ is the degree of $f_{i-1}$ in $x_{r}$. Since char $(R)=0$, we have $f_{i-1}\left(a_{1}, \ldots, a_{r}\right)=0$; thus $f_{i-1}$ vanishes on $S$. This proves inductively that $f$ vanishes on $S$.

Lemma 2. Suppose every semisimple $\mathscr{F}$-ring is commutative. If $A$ is an algebra over a field $F$ and satisfies any of the following conditions, then $C(A)$ is nil:

(i) $A$ is an $\mathscr{F}$-ring with $\mathscr{F}$ finite;

(ii) $A$ is an $\mathscr{F}$-ring with $\mathscr{F}$ linear;

(iii) $A$ is an $\mathscr{F}^{+}$-ring and $\operatorname{char}(A)=0$.

Proof. Let $c \in C(A)$. Then

$$
c=\sum_{i=1}^{m}\left(q_{i} z_{i}+a_{i} z_{i}+z_{i} b_{i}+r_{i} z_{i} s_{i}\right),
$$


where the $q_{i}$ are integers, the $a_{i}, b_{i}, r_{i}, s_{i}, x_{i}, y_{i}$ are in $A$, and $z_{i}=\left[x_{i}, y_{i}\right]$. Let $A_{0}$ be the subalgebra of $A$ generated by all $a_{i}, b_{i}, r_{i}, s_{i}, x_{i}, y_{i}$. If (i) holds, then $A_{0}$ is a finitely generated algebra satisfying a polynomial identity, and hence $J\left(A_{0}\right)$ is a nil ideal [1]. But $A_{0} / J\left(A_{0}\right)$ is a semisimple $\mathscr{F}$-ring and hence commutative by hypothesis. Thus $c \in C\left(A_{0}\right) \subseteq J\left(A_{0}\right)$ which shows that $c$ is nilpotent.

In cases (ii) and (iii) let $K$ be an uncountable extension field of $F$, and consider the tensor product algebra $A_{0} \otimes_{F} K$ over $K$. If (ii) holds, then $A_{0} \otimes_{F} K$ inherits the $\mathscr{F}$-ring property. If (iii) holds, then $A_{0}$ is an $\mathscr{F}^{+}$-ring and hence by (i) of Lemma 1 an $\hat{\mathscr{F}}$-ring. In this case $A_{0} \otimes_{F} K$ is also an $\hat{\mathscr{F}}$-ring and by (ii) of Lemma 1, $A_{0} \bigotimes_{F} K$ is an $\mathscr{F}^{+}$-ring. Thus if $A$ satisfies one of conditions (ii) and (iii), then $A_{0} \otimes_{F} K$ satisfies the same condition. Now $A_{0} \otimes_{F} K$ is a finitely generated algebra over an uncountable field and hence $J\left(A_{0} \otimes_{F} \dot{K}\right)$ is nil [2]. But, just as in case (i), $A_{0} \otimes_{F} K / J\left(A_{0} \otimes_{F} K\right)$ is a semisimple $\mathscr{F}$-ring and hence commutative. Thus $C\left(A_{0} \otimes_{F} K\right)$ is nil, whence so is $C\left(A_{0}\right)$ and since $c \in C\left(A_{0}\right), c$ is nilpotent.

A special case (Lemma 3 below) of a lemma due to Amitsur (cf. [6]) yields the results stated in Lemma 2 for arbitrary rings as well as for algebras over fields. Lemma 3 also appears in [8]. Suppose $\operatorname{char}(R)=0$, and let $M=\{(x, n) \mid x \in R ; n \neq 0$, an integer $\}$. Given $\left(x_{1}, n_{1}\right),\left(x_{2}, n_{2}\right)$ in $M$ define $\left(x_{1}, n_{1}\right) \sim\left(x_{2}, n_{2}\right)$ if and only if $n_{2} x_{1}=n_{1} x_{2}$. This defines an equivalence relation on $M$; let $R^{*}$ be the set of equivalence classes. Denote the equivalence class of $(x, n)$ by $[x, n]$; in $R^{*}$ define addition and multiplication by

$$
\left[x_{1}, n_{1}\right]+\left[x_{2}, n_{2}\right]=\left[n_{2} x_{1}+n_{1} x_{2}, n_{1} n_{2}\right] \text { and }\left[x_{1}, n_{1}\right] \cdot\left[x_{2}, n_{2}\right]=\left[x_{1} x_{2}, n_{1} n_{2}\right] \text {. }
$$

$R^{*}$ is then a ring, in fact an algebra over the field of rational numbers. Also $R$ can be embedded in $R^{*}$ by means of the isomorphism $a \rightarrow[a, 1], a \in R$.

Lemma (AMITSUR). Let $\mathscr{R}$ be a class of rings, and suppose that for each $R$ in $\mathscr{R}$ there is given a subset $Q(R) \subseteq R$. Suppose further that

(i) for every $R$ in $\mathscr{R}$ and every homomorphism $\theta$ of $R, R \theta$ belongs to $\mathscr{R}$ and $Q(R) \theta \subseteq Q(R \theta)$;

(ii) $Q(R)$ is nil for all $R$ in $\mathscr{R}$ whose characteristic is either 0 or a prime.

Then $Q(R)$ is nil for every $R$ in $\mathscr{R}$.

LeMma 3. Let $P$ be a property defined on rings such that

(1) if $P(R)$ is true, then so is $P(R / U)$ for every ideal $U$ of $R$;

(2) if char $(R)=0$ and $P(R)$ is true, then so is $P\left(R^{*}\right)$;

(3) if $A$ is an algebra over a field for which $P(A)$ is true, then $C(A)$ is nil.

Then for any ring $R$, if $P(R)$ is true, $C(R)$ is nil.

Proof. Let $\mathscr{R}$ be the class of rings $R$ with $P(R)$ true and let $Q(R)=C(R)$. Then (i) of Amitsur's lemma clearly holds (using (1)). Thus it is only necessary to check Amitsur's condition (ii). In characteristic $0, P(R)$ gives $P\left(R^{*}\right)$ by (2), and hence $C\left(R^{*}\right)$ is nil by (3), whence $C(R)$, which is embedded in $C\left(R^{*}\right)$, is also nil. In characteristic $p$, of course $R$ is already an algebra, and so $C(R)$ is nil by (3). 
Lemma 4. Suppose char $(R)=0$. Then

(i) if $R$ is an $\mathscr{F}$-ring, so is $R^{*}$;

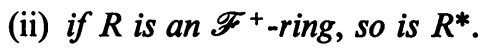

Proof. Suppose first that $R$ is an $\mathscr{F}$-ring, and let $S^{*}$ be a finite subset of $R^{*}$. For each $s^{*} \in S^{*}$ choose one $a \in R$ for which there exists an integer $n \neq 0$ such that $s^{*}=[a, n]$, and let $S$ be the (finite) set of $a$ 's chosen. There exists $f \in \mathscr{F}$ which vanishes on $S$. Suppose $f$ is a polynomial in $k$ indeterminates $x_{1}, \ldots, x_{k}$, of degree $d_{i}$ in $x_{i}$; choose any $k$ elements $\left[a_{1}, n_{1}\right], \ldots,\left[a_{k}, n_{k}\right]$ in $S$ (where $a_{1}, \ldots, a_{k}$ are in $S)$. Then $f\left(\left[a_{1}, n_{1}\right], \ldots,\left[a_{k}, n_{k}\right]\right)=\left[f\left(a_{1}, \ldots, a_{k}\right), n_{1}^{d} \cdots n_{k}^{d_{k}}\right]=\left[0, n_{1}^{d_{1}} \cdots n_{k^{k}}^{d}\right]=0^{*}$, denoting the zero element of $R^{*}$ by $0^{*}$. Hence $f$ vanishes on $S^{*}$ which proves that $R^{*}$ is an $\mathscr{F}$-ring.

Now suppose that $R$ is an $\mathscr{F}^{+}$-ring, and let $S^{*}$ be a finitely generated additive subgroup of $R^{*}$. Let $T^{*}$ be a (finite) set of generators of $S^{*}$, and for each $t^{*} \in T^{*}$ choose one $a \in R$ for which there exists an integer $n \neq 0$ such that $[a, n]=t^{*}$. Let $T$ be the (finite) set of $a$ 's chosen, and let $S$ be the additive subgroup of $R$ generated by $T$. There exists $f \in \mathscr{F}$ which vanishes on $S$. Suppose $f$ is a polynomial in $k$ indeterminates $x_{1}, \ldots, x_{k}$ and of total degree $d$; choose $s_{1}^{*}, \ldots, s_{k}^{*}$ in $S^{*}$. There exist $a_{1}, \ldots, a_{r}$ in $T$, nonzero integers $n_{1}, \ldots, n_{r}$, and sequences $\left\{q_{i}^{(j)}\right\}_{i=1}^{r}(1 \leqq j \leqq k)$ of integers such that

$$
s_{j}^{*}=\sum_{i=1}^{r} q_{i}^{(j)}\left[a_{i}, n_{i}\right] \quad(1 \leqq j \leqq k) .
$$

Then (where $\hat{n}_{i}$ denotes omission of $n_{i}$ )

$$
\begin{aligned}
f\left(s_{1}^{*}, \ldots,\right. & \left.s_{k}^{*}\right) \\
& =f\left(\sum\left[q_{i}^{(1)} a_{i}, n_{i}\right], \ldots, \sum\left[q_{i}^{(k)} a_{i}, n_{i}\right]\right) \\
& =f\left(\left[\sum q_{i}^{(1)} n_{1} \cdots \hat{n}_{i} \cdots n_{r} a_{i}, n_{1} \cdots n_{r}\right], \ldots,\left[\sum q_{i}^{(k)} n_{1} \cdots \hat{n}_{i} \cdots n_{r} a_{i}, n_{1} \cdots n_{r}\right]\right) \\
& =\left[f\left(\sum q_{i}^{(1)} n_{1} \cdots \hat{n}_{i} \cdots n_{r} a_{i}, \ldots, \sum q_{i}^{(k)} n_{1} \cdots \hat{n}_{i} \cdots n_{r} a_{i}\right), n_{1}^{d} \cdots n_{r}^{d}\right] \\
& =\left[0, n_{1}^{d} \cdots n_{r}^{d}\right] \\
& =0^{*} .
\end{aligned}
$$

Hence $f$ vanishes on $S^{*}$ which shows that $R^{*}$ is an $\mathscr{F}^{+}$-ring.

THEOREM 1. Suppose every semisimple $\mathscr{F}$-ring is commutative. If $R$ satisfies any of the following, then $C(R)$ is nil:

(i) $R$ is an $\mathscr{F}$-ring with $\mathscr{F}$ finite;

(ii) $R$ is an $\mathscr{F}$-ring with $\mathscr{F}$ linear;

(iii) $R$ is an $\mathscr{F}^{+}$-ring and char $(R)=0$.

Proof. In cases (i) and (ii) let $P$ be the property of being an $\mathscr{F}$-ring. Clearly, (1) of Lemma 3 holds, Lemma 4 yields (2), and (3) follows from Lemma 2. In case (iii) we have that $R^{*}$ is an $\mathscr{F}^{+}$-ring which is also an algebra over a field so that 
from (iii) of Lemma 2 we see that $C\left(R^{*}\right)$ is nil. Since $R$ is embedded in $R^{*}$, $C(R) \subseteq C\left(R^{*}\right)$ is also nil.

At this point perhaps it should be mentioned that, although our main concern is with the commutator ideal $C(R)$, most of the preceding results (in fact all except (i) of Lemma 2 and (i) of Theorem 1) go through for any ideal (or subset) $Q(R)$ satisfying $Q(R) \theta \subseteq Q(R \theta), Q(R)^{*} \subseteq Q\left(R^{*}\right)$, and $Q(A) \otimes K \subseteq Q(A \otimes K)$.

We now give several applications of the foregoing theorems. An unsolved problem in ring theory is the following: If the commutators in a ring are nilpotent, is the commutator ideal nil? The answer is known to be yes when the index of nilpotence is bounded [7, p. 29], [14]. The next theorem, at least in the characteristic 0 case, gives a result which is intermediate to the bounded index case and the most general case.

THEOREM 2. Suppose char $(R)=0$. If for every finitely generated additive subgroup $S$ of $R$ there exists a positive integer $n$ such that $(x y-y x)^{n}=0$ for all $x, y$ in $S$, then $C(R)$ is nil.

Proof. Let $\mathscr{F}$ be the set of polynomials $(x y-y x)^{n}(n=1,2, \ldots)$. Then $R$ is an $\mathscr{F}^{+}$-ring, and since it is known that every semisimple $\mathscr{F}$-ring is commutative, (iii) of Theorem 1 applies.

For $x, y$ in $R$ define $e_{1}(x, y)=[x, y]$ and $e_{k}(x, y)=\left[e_{k-1}(x, y), y\right]$ for $k>1 . R$ is called a $K$-ring if and only if for every $x, y$ in $R$ there exist integers $k=k(x, y)$ and $n=n(x, y)$ such that $e_{k}\left(x, y^{n}\right)=0 . K$-rings have been studied in [6] and [12]. In [12] it is proved that every $K$-ring with $k$ and $n$ independent of $y$ has nil commutator ideal. The following gives an alternate proof of this result, and, in fact, a more general theorem.

THEOREM 3. Suppose that for every $x \in R$ and every finitely generated additive subgroup $S$ of $R$ there exist integers $k=k(x, S)$ and $n=n(x, S)$ such that $e_{k}\left(x, y^{n}\right)=0$ for every $y \in S$. Then $C(R)$ is nil.

Proof. Let $\mathscr{F}$ be the set of polynomials $e_{k}\left(x, y^{n}\right)$ for all positive integers $k$ and $n$, where $x$ and $y$ are indeterminates. We first show that $R$ is an $\mathscr{F}^{+}$-ring. Let $S$ be a finitely generated additive subgroup of $R$, and let $T$ be a finite set of generators of $S$. For each $a \in T$ there exist integers $k=k(a, S)$ and $n=n(a, S)$ such that $e_{k}\left(a, b^{n}\right)=0$ for every $b \in S$. Let

$$
k=\max \{k(a, S) \mid a \in T\}, \quad n=\prod\{n(a, S) \mid a \in T\} .
$$

Then $e_{k}\left(a, b^{n}\right)=0$ for every generator $a \in T$ and every $b \in S$ [12, Lemma 3]. But the polynomial $e_{k}\left(x, y^{n}\right)$ is linear in $x$; hence, $e_{k}\left(a, b^{n}\right)=0$ for all $a, b \in S$. Therefore $R$ is an $\mathscr{F}^{+}$-ring. Drazin has proved [6] that

(A) every $K$-ring of prime characteristic has nil commutator ideal,

(B) every semisimple $K$-ring is commutative.

Upon taking $\mathscr{R}$ to be the class of $\mathscr{F}^{+}$-rings, we see from Amitsur's lemma that it 
suffices to consider only the cases of prime and zero characteristic. (A) above takes care of the former, while in the latter case, (iii) of Theorem 1 and (B) yield the desired conclusion.

We now prove two theorems, one having to do with the case in which $\mathscr{F}$ is linear and the other with that in which $\mathscr{F}$ is finite, which give necessary and sufficient conditions for every $\mathscr{F}$-ring to have nil commutator ideal.

THEOREM 4. Suppose $\mathscr{F}$ is linear. Then the following are equivalent:

(i) every $\mathscr{F}$-ring has nil commutator ideal;

(ii) for every prime $p$ the ring $G F(p)_{2}$ of 2 by 2 matrices over $G F(p)$ is not an $\mathscr{F}$-ring.

Proof. If (i) holds and $G F(p)_{2}$ is an $\mathscr{F}$-ring for some prime $p$, then $G F(p)_{2}$ is a simple, nonnil, noncommutative ring whose commutator ideal is nil, an impossibility. Thus (i) implies (ii).

Assume (ii) holds, and let $R$ be an $\mathscr{F}$-ring.

Case I: $R$ is a primitive ring. There exists a division ring $\Delta$ such that either $R \approx \Delta_{n}$ for some positive integer $n$, or for each positive integer $m$ there is a subring $S_{m}$ of $R$ and a homomorphism $\phi_{m}$ of $S_{m}$ onto $\Delta_{m}$ [10, p. 33]. If $R \not \Delta \Delta$, then $\Delta_{2}$ inherits the property of being an $\mathscr{F}$-ring. This contradicts (ii) in case char $(\Delta)=p \neq 0$, so assume char $(\Delta)=0$. Then $\Delta$ contains a subring $J$ isomorphic to the ring of integers. The ring $J_{2}$, as a subring of $\Delta_{2}$, is then an $\mathscr{F}$-ring. But then for any prime $p$, the ring $G F(p)_{2}$, as a homomorphic image of $J_{2}$ (under a natural homomorphism) is an $\mathscr{F}$-ring, a contradiction. Thus $R \approx \Delta$, and hence every primitive $\mathscr{F}$-ring is a division ring.

Case II: $R$ is a division ring. Suppose $R$ is noncommutative and let $K \supset Z$ be a maximal subfield of $R$, where $Z$ is the center of $R$. By a theorem of Nakayama and Azumaya [7, p. 108], [15] $R \otimes_{\mathrm{Z}} K$ is a dense ring of linear transformations on $R$ as a vector space over $K$. But $R \otimes_{z} K$ is an $\mathscr{F}$-ring since $\mathscr{F}$ is linear, and hence by Case I we have $R \otimes_{z} K \approx K$, and since $R$ is embedded in $R \otimes_{z} K, R$ is commutative.

Therefore by Case II every division $\mathscr{F}$-ring is commutative, and by Case I every primitive $\mathscr{F}$-ring is a division ring and hence commutative.

Case III: $R$ is a semisimple ring. $R$ is a subdirect sum of primitive (and hence commutative) $\mathscr{F}$-rings; thus $R$ is commutative.

Case IV: $R$ is any $\mathscr{F}$-ring. Since every semisimple $\mathscr{F}$-ring is commutative and $\mathscr{F}$ is linear, (ii) of Theorem 1 applies.

THeOREM 5. Suppose that $\mathscr{F}$ is finite. Then (i) and (ii) of Theorem 4 are equivalent.

Proof. (i) implies (ii) as in Theorem 4. Assume (ii) holds. The proof that every primitive $\mathscr{F}$-ring is a division ring is identical with Case I of the proof of Theorem 4. In Case II, since $R$ satisfies a polynomial identity, $R$ is finite-dimensional over $Z$ [11]. The dimension is, in fact, a square, say $[R: Z]=n^{2}$. Furthermore $[R: K]=n$, and $R \otimes_{\mathrm{Z}} K$, as a dense ring of linear transformations on $R$ over $K$, 
is isomorphic to $K_{n}$. If $Z$ is finite, then $R$, as a finite-dimensional vector space over a finite field, is a finite division ring and hence commutative by Wedderburn's Theorem. If $Z$ is infinite, then it follows from a lemma of Amitsur [3] that $R$ and $R \otimes_{z} K$ satisfy precisely the same polynomial identities. But then $K_{n} \approx R \otimes_{z} K$ is an $\mathscr{F}$-ring. Unless $n=1$, it follows, just as in Case $I$ of the proof of Theorem 4, that $G F(p)_{2}$ is an $\mathscr{F}$-ring for some prime $p$, contradicting (ii). Thus $n=1$ and $R$ is commutative. The semisimple case now follows as in Theorem 4 , and the general case reduces to (i) of Theorem 1.

We conclude with several applications of Theorems 4 and 5.

THEOREM 6. If the commutators of $R$ commute, then $C(R)$ is nil.

Proof. Let $\mathscr{F}$ consist of the single polynomial

$$
f\left(x_{1}, x_{2}, x_{3}, x_{4}\right)=\left[x_{1}, x_{2}\right]\left[x_{3}, x_{4}\right]-\left[x_{3}, x_{4}\right]\left[x_{1}, x_{2}\right] .
$$

To prove the theorem we verify (ii) of Theorem 4 (or Theorem 5). This is easily done by taking

$$
x_{1}=\left(\begin{array}{ll}
0 & 1 \\
0 & 0
\end{array}\right), \quad x_{2}=x_{4}=\left(\begin{array}{ll}
1 & 0 \\
0 & 0
\end{array}\right), \quad x_{3}=\left(\begin{array}{ll}
0 & 0 \\
1 & 0
\end{array}\right)
$$

COROllary. A division ring is commutative if its commutators commute.

More generally, using Theorem 5, we have

THEOREM 7. If there exist fixed positive integers $k$ and $n$ such that all elements of the form $e_{k}\left(x, y^{n}\right)$ commute, then $C(R)$ is nil.

Another consequence of Theorem 5 is a result which was proved in [13], namely,

THEOREM 8. If there exist fixed positive integers $k, n$, and $q$ such that $\left(e_{k}\left(x, y^{n}\right)\right)^{q}=0$ for all $x, y$ in $R$, then $C(R)$ is nil.

In [9] Herstein considered, in turn, rings satisfying the identities $(x y)^{n}=x^{n} y^{n}$ and $(x+y)^{n}=x^{n}+y^{n}$ for some fixed $n>1$ and showed that in each case the commutator ideal is nil. One could attempt generalizations of these theorems in several ways; for example, one could (a) let $n$ depend on $x$ and $y$ or (b) assume the identity holds, not for all elements $x$ and $y$, but only for certain elements (e.g., commutators). The preceding theorems do not seem to yield an answer for (a); however, (b) can be handled by Theorem 5. For that matter one could consider rings satisfying any identity (e.g., $(x y)^{n}=y^{n} x^{n}$ for commutators $x$ and $y$ ) not satisfied by the ring of $2 \times 2$ matrices over the prime field with $p$ elements ( $p$ any prime) and conclude that the commutator ideal is nil.

\section{REFERENCES}

1. S. A. Amitsur, A generalization of Hilbert's Nullstellensatz, Proc. Amer. Math. Soc. 8 (1957), 649-656.

2. - Algebras over infinite fields, Proc. Amer. Math. Soc. 7 (1956), 35-48. 
3. - The identities of PI-rings, Proc. Amer. Math. Soc. 4 (1953), 27-34.

4. R. Brauer, On a theorem of H. Cartan, Bull. Amer. Math. Soc. 55 (1949), 619-620.

5. M. P. Drazin, Rings with nil commutator ideals, Rend. Circ. Mat. Palermo (2) 6 (1957), 51-64.

6. - Engel rings and a result of Herstein and Kaplansky, Amer. J. Math. 77 (1955), 895-913.

7. I. N. Herstein, Theory of rings, University of Chicago Lecture Notes, Chicago, Ill., 1961.

8. _- A remark on rings and algebras, Michigan Math. J. 10 (1963), 269-272.

9. —- Power maps in rings, Michigan Math. J. 8 (1961), 29-32.

10. N. Jacobson, Structure of rings, Amer. Math. Soc. Colloq. Publ. Vol. 37, Amer. Math. Soc., Providence, R. I., 1956; revised, 1964.

11. I. Kaplansky, Rings with a polynomial identity, Bull. Amer. Math. Soc. 54 (1948), 575-580.

12. T. P. Kezlan, Some rings with nil commutator ideals, Michigan Math. J. 12 (1965), 105-111.

13. - A note on higher commutators of bounded nilpotence, Amer. Math. Monthly 73 (1966), 632-633.

14. E. Kleinfeld, Simple alternative rings, Ann. of Math. 58 (1953), 544-547.

15. T. Nakayama and G. Azumaya, Über einfache distributive Systeme unendlicher Ränge. II, Proc. Imp. Acad. Tokyo 20 (1944), 348-352.

UNIVERSITY OF TEXAS, Austin, Texas 